\title{
CONTINUITY OF NONLINEAR MONOTONE OPERATORS
}

\author{
S. P. FITZPATRICK ${ }^{1,2}$
}

\begin{abstract}
If a Banach space $E$ has an equivalent norm such that weak* sequential convergence and norm convergence coincide on the dual unit sphere, then every monotone operator on $E$ is single-valued and norm-norm continuous on a dense $G_{\delta}$ subset of $E$. In particular, this holds for reflexive spaces.
\end{abstract}

Let $E$ be a real Banach space with dual $E^{*}$. A multivalued mapping $T: E \rightarrow E^{*}$ is called a monotone operator if $\left\langle x^{*}-y^{*}, x-y \geqslant 0\right\rangle$ whenever $x^{*} \in T x$ and $y^{*} \in T y$. It is called maximal monotone if, in addition, its graph, $\left\{\left(x, x^{*}\right): x \in E, x^{*} \in T x\right\}$, is not properly contained in the graph of any monotone operator on $E$.

We say that a monotone operator $T: E \rightarrow E^{*}$ is locally bounded at $x \in E$ if there is a neighborhood $U$ of $x$ such that $T(U)=\cup\{T y: y \in U\}$ is a bounded subset of $E^{*}$. This does not demand that $x \in D(T)=\{y \in E: T y$ $\neq \varnothing\}$. We say that $T$ is continuous at a point $x \in D(T)$ if, whenever $x_{n} \rightarrow x, x_{n}^{*} \in T x_{n}$ and $x^{*} \in T x$, we have $\left\|x_{n}^{*}-x^{*}\right\| \rightarrow 0$. If $T$ is continuous at $x$, then it is necessarily single-valued at $x$, that is, $T x$ has exactly one element.

We will assume from now on that $T$ is a maximal monotone operator on $E$, with $D(T)=E$. This latter hypothesis, while not strictly necessary, simplifies both the statements and the proofs of our results. All the proofs can actually be extended to the case where $D(T) \neq E$, provided int conv $D(T) \neq \varnothing$. The reason for this stems from the first part of the following result of Rockafellar [9], which will be of further use to us.

Proposition A. Let $T: E \rightarrow E^{*}$ be a maximal monotone operator with int conv $D(T) \neq \varnothing$. Then int $D(T)$ is convex, $\operatorname{cl} D(T)=\mathrm{cl}$ int $D(T), T$ is locally bounded at each point of int $D(T)$ and $T$ is not locally bounded at any point of the boundary of $D(T)$.

The motivation for studying monotone operators comes from the study of

Received by the editors May 11, 1976.

AMS (MOS) subject classifications (1970). Primary $47 \mathrm{H} 05$.

1 This work was done under the supervision of Professor R. R. Phelps while the author was a Ph.D. student at the University of Washington. It was supported by a Commonwealth Scientific and Industrial Research Organization Postgraduate Studentship.

2 The author notes that the same result has recently been announced by $P$. Kenderov and $R$. Robert, in C. R. Acad. Sci. Paris 282 (1976), No. 16. 
integral and partial differential equations, and from the theory of convex functions. The subdifferential $\partial f$ of a lower semicontinuous convex function $f: E \rightarrow \mathbf{R}$, where

$$
\partial f(x)=\left\{x^{*} \in E^{*}:\left\langle x^{*}, x-y\right\rangle \geqslant f(x)-f(y), \text { all } y \in E\right\}
$$

is a maximal monotone operator (Rockafellar [10]; later, Taylor [11] gave an easier proof), and maximal monotone operators which are not the subdifferentials of lower semicontinuous convex functions (that is, are not cyclically monotone [10]) have some of the desirable properties of those which are. For instance, if $E$ is separable (Zarantonello [12]), or if $E^{*}$ has an equivalent strictly convex dual norm (Kenderov [5]), then the set $S(T)$ of points where $T$ is single-valued is a dense $G_{\delta}$ subset of $E$, hence topologically "almost all" of $E$. In the finite dimensional case, Zarantonello showed that $S(T)$ is almost all of $E$ with respect to Lebesgue measure, too. Using Aronszajn's generalization of the notion of sets of measure zero [1], this result remains valid for separable spaces. These results generalize known results about lower semicontinuous convex functions $f$ on $E$, for $f$ is Gâteaux (resp. Fréchet) differentiable at $x$ if and only if $\partial f$ is single-valued (resp. continuous) at $x$.

As in Asplund [2] and Namioka and Phelps [6], we define $E$ to be a weak differentiability space (resp. Asplund space) if every continuous convex function $f: E \rightarrow \mathbf{R}$ is Gâteaux (resp. Fréchet) differentiable on a dense $G_{\delta}$ subset of $E$.

We now ask

Question (A). If $E$ is an Asplund space, is $T$ necessarily continuous on a dense $G_{\delta}$ subset of $E$ ?

Question (B). If $E$ is a weak differentiability space, is $T$ necessarily singlevalued on a dense $G_{\delta}$ subset of $E$ ?

The results of Kenderov and Zarantonello answer Question (B) affirmatively for most of the known weak differentiability spaces, but the general case for both questions seems difficult.

Our main result is the following partial answer to Question (A).

THEOREM 1. Let $E$ be a Banach space which admits an equivalent norm whose dual norm satisfies $\left(\mathrm{H}^{*}\right)$ :

$$
\begin{aligned}
& \text { If } x_{n}^{*} \in E^{*}, x_{n}^{*} \rightarrow x_{0}^{*} \text { weak }^{*} \text { and }\left\|x_{n}^{*}\right\| \rightarrow\left\|x_{0}^{*}\right\|, \\
& \text { then }\left\|x_{n}^{*}-x_{0}^{*}\right\| \rightarrow 0 .
\end{aligned}
$$

Then for any maximal monotone operator $T: E \rightarrow E^{*}$, the set $C(T)=\{x$ $\in E: T$ is continuous at $x\}$ is a dense $G_{\delta}$ subset of $E$.

We know of no Asplund spaces whose duals are not locally uniformly convex for some equivalent dual norm, and it is easy to see that if a dual norm on $E^{*}$ is locally uniformly convex, then it satisfies $\left(H^{*}\right)$. We also note that $\left(H^{*}\right)$ is apparently weaker then $(* *)$ of Corollary 8 of Namioka and Phelps [6], 
so we thus have a very different proof of that corollary.

If $E$ is finite dimensional, then continuity follows from single-valuedness, and Zarantonello [12] shows that the points of continuity are almost all of $E$ with respect to Lebesgue measure. Robert [7], [8], has proved Theorem 1 for separable spaces with separable duals. Theorem 1 covers all reflexive spaces, of course.

Now we turn to the proof of the theorem. We do not assume $\left(\mathrm{H}^{*}\right)$ until Lemma 7; before that $E$ can be any Banach space.

The following proposition was announced by Kenderov; we prove it for the sake of completeness.

Proposition B. If $T$ is a maximal monotone operator and $x \in E$, then given a weak* neighborhood $W$ of $T x$, there is a neighborhood $U$ of $x$ such that $T(U) \subset W$.

Proof. Suppose $x_{n} \rightarrow x$ and $x_{n}^{*} \in T x_{n}$. Then by local boundedness (Proposition A) we may assume that $x_{n}^{*} \rightarrow x^{*} \in E^{*}$, weak*. We show that $x^{*} \in T x$ : to do this, let $y \in E$ and $y^{*} \in T y$. Then

$$
0 \leqslant\left\langle x_{n}^{*}-y^{*}, x_{n}-y\right\rangle=\left\langle x_{n}^{*}-y^{*}, x-y\right\rangle+\left\langle x_{n}^{*}-y^{*}, x_{n}-x\right\rangle
$$

and in the limit we get $\left\langle x^{*}-y^{*}, x-y\right\rangle \geqslant 0$, so $x^{*} \in T x$ by maximality. This is sufficient to prove the proposition.

The next result is due to Browder [3].

Proposition C. If $T$ is maximal monotone and $x \in E$, then $T x$ is a weak* compact convex subset of $E^{*}$.

(We are assuming that $D(T)=E$. Otherwise these would hold only for $x \in \operatorname{int} D(T)$.) The next result was announced by Kenderov [5].

Lemma 2. Let $T$ be maximal monotone and define $f: E \rightarrow \mathbf{R}$ by $f(x)$ $=\inf \left\{\left\|x^{*}\right\|: x^{*} \in T x\right\}$. Then $f$ is lower semicontinuous.

Proof. Let $x_{n} \rightarrow x$, and suppose $\liminf f\left(x_{n}\right)<f(x)$. Then without loss of generality, there are $x_{n}^{*} \in T x_{n}$ and $\alpha>0$ such that $\left\|x_{n}^{*}\right\|<f(x)-\alpha$. Now $\left(x_{n}^{*}\right)$ has a weak* convergent subsequence, by local boundedness (Proposition A), so we can assume that $x_{n}^{*} \rightarrow x^{*}$ weak*. By maximality $x^{*} \in T x$. Since the norm in $E^{*}$ is weak* lower semicontinuous, $\left\|x^{*}\right\| \leqslant \liminf \left\|x_{n}^{*}\right\| \leqslant f(x)-\alpha$, which is a contradiction.

By a Baire category result (cf. e.g. [4, Corollary 7.6]) we immediately obtain

Corollary 3. The set $A(T)$ where $f$ is continuous is a dense $G_{\delta}$ subset of $E$.

The following lemma contains the critical point in our reconstruction of the argument in Kenderov [5].

Lemma 4. If $T$ is maximal monotone, $x \in A(T)$ and $x^{*} \in T x$, then $\left\|x^{*}\right\|$ $=f(x)$. 
Proof. If $\left\|x^{*}\right\|>\dot{f}(x)$, then choose $z \in E,\|z\|=1$, such that $\left\langle x^{*}, z\right\rangle$ $>f(x)$. Now by continuity of $f$ at $x$, for $\varepsilon>0$ small enough there exists $x_{0}^{*} \in T(x+\varepsilon z)$ such that $\left\|x_{0}^{*}\right\|\left\langle\left\langle x^{*}, z\right\rangle\right.$. But $\left\langle x_{0}^{*}-x^{*},(x+\varepsilon z)-x\right\rangle \geqslant 0$ by monotonicity, so $\left.\left\langle x_{0}^{*}, z\right\rangle \geqslant\left\langle x^{*}, z\right\rangle\right\rangle\left\|x_{0}^{*}\right\|$, contradicting the fact that $\|z\|=1$.

LemMA 5. If $T$ is maximal monotone, $x_{0} \in A(T)$ and $x_{n} \rightarrow x_{0}$, then $\left\|x_{n}^{*}\right\|$ $\rightarrow\left\|x_{0}^{*}\right\|$, whenever $x_{n}^{*} \in T x_{n}$ and $x_{0}^{*} \in T x_{0}$.

Proof. Suppose limsup $\left\|x_{n}^{*}\right\|>\left\|x_{0}^{*}\right\|+\alpha$, where $\alpha>0$, and choose a subsequence, again called $\left(x_{n}^{*}\right)$, such that $\left\|x_{n}^{*}\right\|>\left\|x_{0}^{*}\right\|+\alpha$. Let $z_{n} \in E$, $\left\|z_{n}\right\|=1$, be such that $\left.\left\langle x_{n}^{*}, z_{n}\right\rangle\right\rangle\left\|x_{0}^{*}\right\|+\alpha$. By local boundedness, we may assume that $\left\|x_{n}^{*}\right\|<N$, for all $n \geqslant 1$. Pick $y_{n} \in B\left(z_{n},(n N)^{-1}\right)=\{x: \| x$ $\left.-z_{n} \|<(n N)^{-1}\right\}$ such that $x_{n}+n^{-1} y_{n} \in A(T)$; this is possible since the open set $x_{n}+n^{-1} B\left(z_{n},(n N)^{-1}\right)$ intersects the dense set $A(T)$. Then we compute

$$
\begin{aligned}
\left\langle x_{n}^{*}, y_{n}\right\rangle & =\left\langle x_{n}^{*}, z_{n}\right\rangle-\left\langle x_{n}^{*}, y_{n}-z_{n}\right\rangle \\
& \geqslant\left\langle x_{n}^{*}, z_{n}\right\rangle-\left\|x_{n}^{*}\right\|(n N)^{-1} \geqslant\left\langle x_{n}^{*}, z_{n}\right\rangle-n^{-1} .
\end{aligned}
$$

Let $y_{n}^{*} \in T\left(x_{n}+n^{-1} y_{n}\right)$; from Lemma 4 it follows that

$$
\left\|y_{n}^{*}\right\|=f\left(x_{n}+n^{-1} y_{n}\right) \text {. }
$$

By monotonicity, we have $\left\langle y_{n}^{*}-x_{n}^{*},\left(x_{n}+n^{-1} y_{n}\right)-x_{n}\right\rangle \geqslant 0$, so

$$
\left\langle y_{n}^{*}, y_{n}\right\rangle \geqslant\left\langle x_{n}^{*}, y_{n}\right\rangle \geqslant\left\langle x_{n}^{*}, z_{n}\right\rangle-n^{-1} \text {. }
$$

Thus,

$$
\left.\left\|y_{n}^{*}\right\|\left\|y_{n}\right\| \geqslant\left\langle x_{n}^{*}, z_{n}\right\rangle-n^{-1}\right\rangle\left\|x_{0}^{*}\right\|-\alpha-n^{-1},
$$

while $\left\|y_{n}\right\|<1+(n N)^{-1}$, so $\lim \sup \left\|y_{n}^{*}\right\| \geqslant\left\|x_{0}^{*}\right\|+\alpha$. Now $x_{n}+n^{-1} y_{n}$ $\rightarrow x_{0}$ and $x_{n}+n^{-1} y_{n} \in A(T)$, so $\left\|y_{n}^{*}\right\|=f\left(x_{n}+n^{-1} y_{n}\right) \rightarrow f\left(x_{0}\right)=\left\|x_{0}^{*}\right\|$, since $x_{0} \in A(T)$. This contradiction shows that lim sup $\left\|x_{n}^{*}\right\| \leqslant\left\|x_{0}^{*}\right\|$.

Now we show that $\lim \inf \left\|x_{n}^{*}\right\| \geqslant\left\|x_{0}^{*}\right\|$. Suppose otherwise; then we may assume that $\left\|x_{n}^{*}\right\|<\left\|x_{0}^{*}\right\|-\alpha$, for some $\alpha>0$. There is a weak* convergent subsequence, again called $\left(x_{n}^{*}\right)$, such that $x_{n}^{*} \rightarrow x^{*} \in T x_{0}$ weak $^{*}$, using local boundedness and Proposition B. Since the norm in $E^{*}$ is weak* lower semicontinuous, we have $\lim \inf \left\|x_{n}^{*}\right\| \geqslant\left\|x^{*}\right\|$ and $\left\|x^{*}\right\|=\left\|x_{0}^{*}\right\|$ by Lemma 4. This is again a contradiction, and we conclude that $\left\|x_{n}^{*}\right\| \rightarrow\left\|x_{0}^{*}\right\|$.

Next, we generalize Asplund's Lemma 6 of [2] to the present situation.

LEMMA 6. Let $T$ be a maximal monotone operator. Then the set of points where $T$ is continuous, $C(T)$, is a $G_{\delta}$ subset of $E$.

Proof. For each $n \geqslant 1$, let $U_{n}=\{x \in E$ : there exists $\delta>0$ such that for 
$y, z \in B(x, \delta),\left\|y^{*}-z^{*}\right\|<n^{-1}$, for any $y^{*} \in T y$ and $\left.z^{*} \in T z\right\}$. It is immediate that $C(T) \subseteq \cap U_{n}$ and it is obvious that $U_{n}$ is always open, so we only have to prove that $\cap U_{n} \subseteq C(T)$.

Suppose $x \in \cap U_{n}$. Then there are $\delta_{n}>0$, which we may take decreasing to 0 , such that if $y, z \in B\left(x, \delta_{n}\right), y^{*} \in T y$ and $z^{*} \in T z$, then $\left\|y^{*}-z^{*}\right\|$ $<n^{-1}$. Thus $K_{n}=\operatorname{cl} T\left(B\left(x, \delta_{n}\right)\right)$ has diameter at most $n^{-1}$, and $K_{n+1} \subseteq K_{n}$. By completeness, $\cap K_{n}=\left\{x^{*}\right\}$ for a unique $x^{*} \in E^{*}$. We claim that $x^{*} \in T x_{n}$; to this end, let $y \in E$ and $y^{*} \in T y$. Then letting $x_{n} \in B\left(x, \delta_{n}\right)$ and $x_{n}^{*} \in T x_{n}$, we have

$$
\begin{aligned}
& \left\langle x^{*}-y^{*}, x-y\right\rangle \\
& \quad=\left\langle x^{*}-x_{n}^{*}, x-y\right\rangle+\left\langle x_{n}^{*}-y^{*}, x_{n}-y\right\rangle+\left\langle x_{n}^{*}-y^{*}, x-x_{n}\right\rangle \\
& \geqslant-n^{-1}\|x-y\|+0-\delta_{n}\left\|x_{n}^{*}-y^{*}\right\| .
\end{aligned}
$$

Since $n$ is arbitrary, we get $\left\langle x^{*}-y^{*}, x-y\right\rangle \geqslant 0$, and hence by maximality, $x^{*} \in T x$. Now if $y \in B\left(x, \delta_{n}\right)$ and $y^{*} \in T y$, then $\left\|y^{*}-x^{*}\right\| \leqslant n^{-1}$. That is, $x \in C(T)$.

We now use property $\left(\mathrm{H}^{*}\right)$ defined earlier.

LEMMA 7. Let $T$ be a maximal monotone operator on a Banach space whose dual has an equivalent dual norm satisfying $\left(\mathrm{H}^{*}\right)$. Suppose $x_{0} \in A(T)$ and $\operatorname{diam}\left(T x_{0}\right)<\varepsilon$. Then there is a neighborhood $V$ of $x$ such that $\operatorname{diam}(T x)<\varepsilon$ for all $x \in V$.

Proof. Suppose that $x_{n} \rightarrow x_{0}$ and $\operatorname{diam}\left(T x_{n}\right) \geqslant \varepsilon$. Take $v_{n}^{*}, w_{n}^{*} \in T x_{n}$ such that $\left\|v_{n}^{*}-w_{n}^{*}\right\| \rightarrow \varepsilon$ as $n \rightarrow \infty$. By local boundedness, we can take a subsequence $\left(v_{n_{j}}^{*}\right)$ of $\left(v_{n}^{*}\right)$ such that $v_{n_{j}}^{*} \rightarrow v^{*}$, weak*. As in the proof of Lemma $5, v^{*} \in T x_{0}$. By Lemma 5 and $\left(\mathrm{H}^{*}\right),\left\|v_{n_{j}}^{*}-v^{*}\right\| \rightarrow 0$. Take a subsequence $\left(w_{m_{j}}^{*}\right)$ of $\left(w_{n_{j}}^{*}\right)$ such that $w_{m_{j}}^{*} \rightarrow w^{*}$ weak*. Then $w^{*} \in T x_{0}$ and $\left\|w_{m_{j}}^{*}-w^{*}\right\| \rightarrow 0$. Now $\left\|v_{m_{j}}^{*}-v^{*}\right\| \rightarrow 0,\left\|w_{m_{j}}^{*}-w^{*}\right\| \rightarrow 0$ and $\left\|v_{m_{j}}^{*}-w_{m_{j}}^{*}\right\|$ $\rightarrow \varepsilon$. Hence $\left\|v^{*}-w^{*}\right\|=\varepsilon$ and $\varepsilon>\operatorname{diam}\left(T x_{0}\right)$, a contradiction.

We now give the proof of Theorem 1 .

Proof. If $y^{*} \in E^{*}$, then $T-y^{*}$ is a maximal monotone operator, where

$$
\left(T-y^{*}\right)(x)=\left\{x^{*}-y^{*}: x^{*} \in T x\right\}, \quad x \in E .
$$

Let $x_{0} \in A(T)$. Then $T x_{0}$ is weak* compact, and $\left\|x^{*}\right\|=\left\|y^{*}\right\|$ for all $x^{*}, y^{*} \in T x_{0}$, so $\left(\mathrm{H}^{*}\right)$ implies that $T x_{0}$ is compact. Thus there is a normdense sequence $\left(y_{m}^{*}\right) \subset T x_{0}$.

Let $A=\bigcap_{m}\left(A(T) \cap A\left(T-y_{m}^{*}\right)\right)$; this is again a dense $G_{\delta}$. Pick $x_{n} \rightarrow x_{0}$, $x_{n} \in A$, and $v_{n}^{*}, w_{n}^{*} \in T x_{n}$ such that $\left\|v_{n}^{*}-w_{n}^{*}\right\|>\frac{1}{2} \operatorname{diam}\left(T x_{n}\right)$. The sequence $\left(v_{n}^{*}\right)$ has a subsequence $\left(v_{n_{j}}^{*}\right)$ converging weak* to $v^{*} \in T x_{0}$, and by $\left(\mathrm{H}^{*}\right)$ and Lemma $5,\left\|v_{n_{j}}^{*}-v^{*}\right\| \rightarrow 0$. By taking $y_{m}^{*}$ close to $v^{*}$, and applying Lemma 4 to $T-y_{m}^{*}$ we see that $\left\|v_{n_{j}}^{*}-v^{*}\right\|=\left\|w_{n_{j}}^{*}-v^{*}\right\|$, since $x_{n_{j}} \in A$. Thus $\left\|v_{n_{j}}^{*}-w_{n_{j}}^{*}\right\| \rightarrow 0$, which means that $\operatorname{diam}\left(T x_{n_{j}}\right) \rightarrow 0$. 
So for every $x_{0} \in A(T)$ and $\varepsilon>0$, there is $z_{0} \in A(T)$ with $\operatorname{diam}\left(T z_{0}\right)<\varepsilon$ and $\left\|x_{0}-z_{0}\right\|<\varepsilon$. Further, by Lemma 7 , there is an open neighborhood $V_{0}$ of $z_{0}$ such that if $x \in V_{0}$ then $\operatorname{diam}(T x)<\varepsilon$. By the density of $A(T)$, we see that $\left\{x \in E: \operatorname{diam}(T x)<n^{-1}\right\}$ contains an open dense set for each $n \geqslant 1$. Thus $S(T)=\{x \in E: \operatorname{diam}(T x)=0\}=\bigcap\left\{x \in E: \operatorname{diam}(T x)<n^{-1}\right\}$ contains a dense $G_{\delta}$. Consequently, $A(T) \cap S(T)$ contains a dense $G_{\delta}$, and $T$ is continuous at each point of the latter set, by Proposition B, Lemma 5 and the property $\left(\mathrm{H}^{*}\right)$. Lemma 6 then gives the theorem.

\section{REFERENCES}

1. N. Aronszajn, Differentiability of Lipschitzian mappings between Banach spaces, Studia Math. (to appear).

2. Edgar Asplund, Fréchet differentiability of convex functions, Acta Math. 121 (1968), 31-47. MR 37 \#6754.

3. F. E. Browder, Multi-valued monotone nonlinear mappings and duality mappings in Banach spaces, Trans. Amer. Math. Soc. 118 (1965), 338-351. MR 31 \# 5114.

4. G. Choquet, Lectures on analysis. Vol. 1, Benjamin, New York, 1969. MR 40 \#3252; erratum, 44, p. 1630.

5. P. S. Kenderov, The set-valued monotone mappings are almost everywhere single-valued, $\mathrm{C} . \mathrm{R}$. Acad. Bulgare Sci. 27 (1974), 1173-1175. MR 50 \# 10913.

6. I. Namioka and R. R. Phelps, Banach spaces which are Asplund spaces, Duke Math. J. 42 (1975), 735-750.

7. R. Robert, Points de continuité des multi-applications semi-continues supérieurement, $\mathbf{C} . \mathbf{R}$. Acad. Sci. Paris 278 (1974), 413-415. MR 50 \#995.

8. Une généralisation aux opérateurs monotones des théorèmes de differentiabilité d'Asplund, Analyse Convexe et Ses Applications, Lecture Notes in Economics and Math. Systems, no. 102, Springer-Verlag, Berlin and New York, 1974, pp. 168-179.

9. R. T. Rockafellar, Local boundedness of nonlinear, monotone operators, Michigan Math. J. 16 (1969), 397-407. MR 40 \#6229.

10. On the maximal monotonicity of subdifferential mappings, Pacific J. Math. 33 (1970), 209-216. MR 41 \# 7432.

11. Peter D. Taylor, Subgradients of a convex function obtained from a directional derivative, Pacific J. Math. 44 (1973), 739-747. MR 48 \#2759.

12. Eduardo H. Zarantonello, Dense single-valuedness of monotone operators, Israel J. Math. 15 (1973), 158-166. MR 48 \#2842.

Department of Mathematics, University of Washington, Seattle, Washington 98195 